\title{
Materials Science Research Hardware for Application on the International Space Station: An Overview of Typical Hardware Requirements and Features
}

\author{
D. A. Schaefer \\ S. Cobb \\ NASA, Marshall Space Flight Center, AL \\ M.R. Fiske \\ Morgan Research Corporation, Huntsville, AL \\ R. Srinivas \\ System Studies \& Simulation, Inc., Huntsville, AL
}

\begin{abstract}
Introduction
NASA's Marshall Space Flight Center (MSFC) is the lead center for Materials Science Microgravity Research. The Materials Science Research Facility (MSRF) is a key development effort underway at MSFC. The MSRF will be the primary facility for microgravity materials science research on board the International Space Station (ISS) and will implement the NASA Materials Science Microgravity Research Program. It will operate in the U.S. Laboratory Module and support U.S. Microgravity Materials Science Investigations. This facility is being designed to maintain the momentum of the U.S. role in microgravity materials science and support NASA's Human Exploration and Development of Space (HEDS) Enterprise goals and objectives for Materials Science.

The MSRF as currently envisioned will consist of three Materials Science
\end{abstract}

Research Racks (MSRR), which will be deployed to the International Space Station (ISS) in phases. Each rack is being designed to accommodate various Experiment Modules, which comprise processing lacilities for peer selected Materials Science experiments. Phased deployment will enable early opportunities for the U.S. and International Partners, and support the timely incorporation of technology updates to the Experiment Modules and sensor devices.

The first Materials Science Research Rack (MSRR-1 will accommodate two Experiment Modules and provide dual operations capability. The initial Materials Science Experiment Module for the first Materials Science Research Rack is an international cooperative activity between NASA's Marshall Space Flight Center and the European Space Agency's (ESA) European Space Research and Technology Center 
(ESTEC). This first International Materials Science payload will consist of the Materials Science Laboratory (MSL) developed by ESA as an Experiment Module. The MSL will provide critical support and control subsystems and will accommodate Module Inserts developed by NASA, ESA, and other International Partners. Module Inserts may be exchanged on-orbit, however, the entire rack must be returned for replacement of the MSL. The second EM is a commercial furnace supplied by MSFC's Space Products Department (SPD) which will be replaced on-orbit.

Architecture studies are currently underway for the follow-on MSRF racks which will optimize the approach to modularity and commonality in the accommodation of planned processing devices, required to satisfy the NASA Material Science Discipline needs. The development of investigation-unique, as well as generic EMs, is planned in order to meet the science requirements of the current and evolving array of Materials Science investigations. This paper will describe the methodology utilized in the definition of science requirements and the conceptual approach for future Experiment Modules to be developed for the Materials Science Research Facility on the International Space Station.

\section{Concept}

The MSRF is scheduled to begin phased deployment with the MSRR-1 on the third Utilization Flight (UF-3) to the ISS. The MSRR-1 will house materials science processing apparatus for satisfying a portion of the near-term and long-range goals and objectives of Materials Science Discipline. Each follow-on Materials Science Research Rack will provide subsystems developed by NASA, and EMs developed by both NASA and International Partners. Each MSRR will accommodate on-orbit replaceable EMs, Module Inserts, investigation-unique apparatus, and/or multi-user generic processing apparatus, and will support a wide variety of investigations. The Experiment Modules for each of the follow-on racks are designed to be "smart" furnaces with the support subsystems designed to accommodate each investigation. The MSRR racks and EMs will provide the optimum flexibility to support on-orbit maintenance and change out of key components in order to accommodate as broad a spectrum of the many materials systems, processing methods, and scientific rescarch thrusts as is practical. The operational life for each rack will be a minimum of live years with a design life of ten years. A preliminary concept for the MSRF compliment of Experiment Modules and Module Inserts is shown in Figure 1.

\section{Figure 1 - Materials Science Research Facility Preliminary Concept}

\section{Science Requirements Definition}

Functional capabilities of each EM and required support subsystem for each MSRR are derived from the science requirements of the current and evolving cadre of Principle Investigators selected through the peer-selected NASA Research Announcement (NRA) 
program. Science requirements for the first rack represent a consolidation of the requirements of the current flight and flight-definition candidate ISS investigations. For MSRR-1, these requirements were used to define a science requirements envelope, which was then used to identify and define various apparatus types, including the Experiment Modules, and Module Inserts. These requirements are documented in the Science Requirements Envelope Document (SRED). The SRED includes specific requirements identified by Principle Investigators (PIs), requirements defined in Science Working Group (SWG) meetings, inputs from Investigator Working Group (IWG) meetings, inputs from Advisory Panels, and identifies key constraints (volume, mass, power, crew time) imposed by ISS.

In order to accommodate the science requirements for investigations that will be identified in the future, a set of "Reference Experiments" are being defined to encompass a wide range of material types, envelope hardware capabilities, and aid in the design of future rack capabilities. These requirements will be documented in a SRED for future hardware. An additional constraint is that these Reference Experiments must be able to accommodate the needs of PIs selected in future NRAs. Other carriers will be utilized for PIs whose requirements can not be satisfied by the envelope capabilities of the MSRF.

The definition of each Reference Experiment is based, largely, on the five basic themes identified as priorities for microgravity materials science research by the NASA Materials Science Discipline Working Group. The themes are as follows:

- Nucleation and Metastable States
- Prediction and Control of
Microstructures
Phase Separation and Interfacial
Phenomena
Transport Phenomena
- Crystal Growth and Defect
Generation

These phenomena will likely be explored in the following classes of materials: Electronic and Photonic materials, Metals and Alloys, Ceramics and Glasses, Polymers, and Composite materials. In addition, each investigation is likely to evaluate more than one theme for a given material. It is expected that the majority of NASA's future microgravity materials science rescarch projects will fall into a matrix of the classes of materials and the themes shown in Figure 2.

\section{Figure 2 - NASA Materials Science Program Research Goals}

A preliminary review of the Reference Experiments indicates many experimentspecific capabilities must be developed into future hardware in order to accommodate all of the requirements imposed by these experiments. For example, many experiments in the future will require imaging techniques that have not traditionally been incorporated into microgravity materials science processing apparatus. Requested capabilities include: 


$\begin{array}{ll}- & \text { Transparent Furnaces } \\ \text { - } & \text { Real-time Digital Video Imaging } \\ \text { - } & \text { Digital X-Ray Camera } \\ \text { - } & \text { Digital/CCD Cameras } \\ \text { - } & \text { Optical Interferometry } \\ \text { - } & \text { Holography } \\ \text { - } & \text { Flow Visualization } \\ \text { - } & \text { Lasers } \\ \text { - } & \text { In-Situ Detection of Defects } \\ \text { - } & \text { Optical Wavelength Imaging } \\ \text { - } & \text { Photo-sensing Devices }\end{array}$

Other capabilities that will require assessment for incorporation into future apparatus development include:

$\begin{array}{ll}\text { - } & \text { Absorption Spectroscopy } \\ \text { - } & \text { Magnetic Fields } \\ \text { - } & \text { Chamber Pressure Control } \\ \text { - } & \text { Thermal Sample Processing } \\ \text { - } & \text { Optical Fiber Thermometry } \\ \text { - } & \text { High Vacuum }\left(10^{-8} \mathrm{mbar}\right)\end{array}$

Sample containment and instrumentation present a new set of challenges and technologies that must be addressed for future microgravity materials processing experiments. The development of Sample Ampoule Cartridge Assemblies (SACAs) for high-temperature processing, toxic sample processing, and new processing methodologies, will require new methods for incorporating instrumentation such as:

- Multiple Thermocouples (up to 20)

- $\quad$ Optical Fiber Thermometry

- $\quad$ Seebeck Measurement

- $\quad$ Sample Resistance Measurement

$\begin{array}{ll}-\quad & \text { Ampoule/Cartridge Failure } \\ \text { - Detection } \\ \text { - Current Pulse Interface } \\ \text { - Demarcation (Peltier Pulsing) } \\ \text { - } \quad \text { Radiation Detection/Shiclding } \\ \text { Spectral Emissivity } \\ \text { Measurement System }\end{array}$

Facility requirements for the rack level subsystems needed to support Experiment Module and Module Insert capabilities are delined by the generic hardware requirements imposed by the appropriate Reference Experiment and investigator-specilic experiment requirements. The requirement definition process, shown in Figure 3, initially results in a set of requirements for the processing apparatus itself as well as for any associated diagnostic equipment. This will also aid in the definition of required support systems (vacuum, gas and/or water servicers, support structures, etc.). The combination of the processing apparatus diagnostic equipment features. and support system capabilities will yield the full MSRF system capabilities.

\section{Figure 3 -Science Requirements Flow into Hardware Capabilities}

\section{Experiment Module Descriptions}

Several Experiment Module (EM) concepts have been developed to satisfy the developing science investigations.

MSRR-1 will initially be installed with the ESA-developed Materials Science Laboratory (MSL) installed on one side of the rack, and a commercial furnace supplied by MSFC's Space Products 
Department (SPD) on the other side. Various Module Inserts are currently in the planning including a Quench Module Insert (QMI) and Diffusion Module Insert (DMI) by NASA and the Low Gradient Furnace Module Insert and Solidification and Quench Furnace (SQF) Module Insert by ESA. Additional follow-on Module Inserts are anticipated by both NASA and our International Partners to satisfy additional investigation objectives.

After the first ISS increment, the SPD furnace will be removed and replaced with a second NASA EM for Materials Science research. This device is planned to be the Pattern Formation and Coarsening Research (PFCR) Apparatus. The PFCR will be a multi-user facility designed to meet the needs of researchers with high-precision, high-stability, lowtemperature requirements. Transparent model materials will be used to study the formation of microstructures and provide an extension of the Spacelab-based Isothermal Dendritic Growth Experiment (IDGE) research. This apparatus will allow isothermal sample melting and undercooling with real-time imaging during solidification instigation.

Specific PFCR hardware features and capabilities will include thermostat-based temperature and pressure control, an operating temperature range of $40^{\circ} \mathrm{C}$ to $70^{\circ} \mathrm{C}$ for long periods of time, highprecision temperature measurement and stability, digital and film photography, and holography. The PFCR will accommodate a number of Isothermal Bath Systems and will offer several technology challenges, particularly in the areas of high-precision temperature measurement and control, and in the design of optical systems.

Another planned EM is the SelfDiffusion in Liquid Elements/Thermophysical Propertics (SDLE/TPP) Module. The objectives of SDLE/TPP are to provide mass and thermal diffusivity measurements over a wide range of temperatures, diffusivity interpretations via diffusivity mechanisms and temperature-dependent liquid structures, and "wall effect" qualification for a varicty of samples. Hardware features and capabilities for the SDLE/TPP EM include multiple diffusivity measurements at different temperatures using only one sample, a sealed, semi-automatic, self-contained EM design, concentration distribution determination of a diflusion sample prior to solidification by radiotracer detection. an operating temperature range up to $1400^{\circ} \mathrm{C}$, and the ability to change out the sample cartridges on-orbit. The EM sample exchange mechanism design is challenging, because the containment of potentially toxic materials must be maintained without disturbing the thermal field.

Additional Experiment Modules planned for development will accommodate PI unique functional and performance requirements such as: Enhanced Peltier Pulsing, Seebeck coefficient measurement, precise furnace translation control and measurement, transparent viewing capability of sample during processing, precise temperature controls and measurement, high gradients, and rapid quench capability. In addition 
these EMs will have automated sample exchange mechanisms and employ additional sensor capability. The design concepts for the EMs will assess the development of Reconfigurable Furnace Modules (RFMs) that can be reconfigured on-orbit. Furnace Translation Systems (FTS) will be designed to provide a full range of translation rate capabilities. Common avionics boxes will be utilized when possible.

One furnace to be developed for followon rack deployment is a high gradient solidification furnace. This Experiment Module would enable crystal growth process studies and also support interfacial energy and flow effect studies as well as interface pattern evolution in alloys. Salient hardware features and capabilities will include: a high gradient furnace module $\left(10-150^{\circ} \mathrm{C} / \mathrm{cm}\right)$ with a reconfigurable gradient zone, an operating temperature range up to $1600^{\circ} \mathrm{C}$, precise furnace translation, control of processing gas, in-situ quench rates up to $100^{\circ} \mathrm{C} / \mathrm{sec}$, and a ten sample automatic sample exchange. Specific challenges associated with the development of this apparatus would include the development of high quench rates which may involve the development of an in-situ quench system, and the establishment of high axial thermal gradients in the sample and associated problems. Bore diameter is anticipated to accommodate sample cartridges of up to $2 \mathrm{~cm}$ maximum diameter

An EM which would provide directional solidification and vapor transport crystal growth processing capabilities for long duration experiments is also desired. Relevant hardware features and capabilities include a processing temperature range up to $1400^{\circ} \mathrm{C}$ with a reconfigurable gradient zone $(1-5 \mathrm{~cm})$, precise furnace translation, automatic sample exchange capability with special optical measurements and real-time video/imaging, in addition to limited Peltier capability. Challenges associated with this apparatus include the requirement of precise translation control.

Another Experiment Module which has been under consideration is a directional solidification furnace with enhanced pulsing capability. This apparatus would be a modified Bridgman furnace with an actively cooled cold zone. It would be used to study processing condition effects in phase selection and interface pattern evolution, and defect generation and control. Key hardware features and capabilities include: an actively cooled cold zone down to $50^{\circ} \mathrm{C}$, with the hot zone at fairly high temperatures (up to $650^{\circ} \mathrm{C}$ ), a furnace module gradient range of $10-100^{\circ} \mathrm{C} / \mathrm{cm}$ with a reconfigurable gradient zone $(1-10 \mathrm{~cm})$, Seebeck voltage measurements, high current/long duration Peltier Pulsing (up to $100 \mathrm{~A}$ for relatively long duration), automatic sample exchange capability, and furnace translation. The primary challenge associated with the development of this EM is the incorporation of the high current Peltier Pulsing capability .

Another potential Experiment Module for future development is a ultra high 
temperature Bridgman furnace. This EM would involve the application of new high temperature materials and sensing devices and would be deployed in latter development stages of the MSRF. The objectives of this furnace are to provide high temperature processing of semiconductor materials and provide isothermal heated zones for diffusion studies including studies of doped silicon. Specific hardware features and capabilities will include a processing temperature range from $1000^{\circ} \mathrm{C}$, to $2300^{\circ} \mathrm{C}$, a high axial thermal gradient $\left(10-100^{\circ} \mathrm{C} / \mathrm{cm}\right)$ in the sample, a reconfigurable gradient zone, isothermal regions in both the hot and cold zones, rapid furnace translation, automatic sample exchange capability, and a capability for automatic shearing of diffusion cells, which would require a drive motor in the sample container. The apparatus will require translation capability of the furnace core with precision control to allow processing of a $20 \mathrm{~cm}$ sample length. The required high temperatures and associated limitations of engineering materials, along with the stringent requirement for isothermality required for diffusion studies present many technical challenges. A design study and early breadboard testing of this high temperature furnace concept are in preliminary stages at the Marshall Space Flight Center. Challenges associated with the development of this apparatus include the requirement for precise translation and the high power requirements associated with a larger bore diameter. Reliability of operation will also be a driver in the design to accommodate the number of high temperature processing timeline runs.
Accommodation of a shear cell motor for the apparatus will be required. The length of the furnace hot zone will be at least $20 \mathrm{~cm}$ to accommodate a sample of up to $20 \mathrm{~cm}$ in length. This Experiment could accommodate variable reconfigurable furnace modules a larger furnace bore diameter of up to $5 \mathrm{~cm}$ in selected configurations.

The phased deployment of follow-on EMs in the second and third MSRRs will allow for numerous enhancements and upgrades. Advanced technology improvements derived from the lurnace technology program will include: higher temperature capabilities, advanced insitu measurement capability, improved failure sensor technology, increased overall thermal performance and improved thermal symmetry and efficiency, improved optical fiber thermometry techniques and additional design features to accommodate ORU scenarios.

\section{Summary}

The Materials Science Research Facility will offer the materials science research community unprecedented opportunitics to perform research in the microgravity environment of space. A time-phased deployment of MSRF racks, each capable of supporting a number of different Experiment Modules, will allow significant flexibility in the design approach to accommodate meaningful basic and applied research for the current and evolving Materials Science investigations. In order to assist in the definition of the science requirements for future Experiment Modules, a set of 
Reference Experiments are being defined to optimize the development of the Experiment Modules and rack accommodations. Based on this activity, preliminary conceptual designs for several fulure EMs are being developed. Many of the required hardware capabilities will present a formidable design challenge relative to compatibility issues, temperature capabilities, sensor technology development and, ultimately, packaging. The development of these facility class Experiment Modules required to support the peer reviewed science investigations through the NRA process will form the foundation for the United States Microgravity Materials Science Research Program and support International cooperative and multidiscipline scientific efforts throughout the life of the International Space Station. This facility class development approach will ultimately provide a technically rewarding and cost effective resource to the science community and the American public. 